\title{
DENNIS R. PAULSON \& MILEN MARINOV (2021) Zootaxa 20th Anniversary Celebration: Odonata section. Zootaxa, 4979: 218-221.
}

Updated Table 2 is presented below.

Originally we had trouble deciding how to compile Table 2, as some of the authors of Zootaxa papers listed affiliation with countries other than their own, but we credited their own country instead. On second thought, we have decided to compile the list based strictly on the credits given in the papers, so that necessitated revising the totals. Thus The Netherlands is very well represented because several authors from other countries list their affiliation with the Naturalis Biodiversity Centre in Leiden. When an author listed affiliation with two countries, as was done in a few cases, we credited both countries in the table.

TABLE 2. Countries of origin of authors of Odonata papers in Zootaxa.
Country of authors
\# papers
Country of authors
\# papers

$\begin{array}{lc}\text { Netherlands } & 75 \\ \text { Brazil } & 61 \\ \text { USA } & 50 \\ \text { China } & 49 \\ \text { Mexico } & 32 \\ \text { France } & 27 \\ \text { Germany } & 23 \\ \text { Australia } & 18 \\ \text { Vietnam } & 18 \\ \text { Argentina } & 17 \\ \text { Malaysia } & 17 \\ \text { Russia } & 16 \\ \text { Colombia } & 15 \\ \text { India } & 14 \\ \text { Thailand } & 12 \\ \text { Japan } & 11 \\ \text { UK } & 10 \\ \text { Spain } & 7 \\ \text { Costa Rica } & 6 \\ \text { Cuba } & 5 \\ \text { New Zealand } & 5 \\ \text { Portugal } & 4\end{array}$

$\begin{aligned} \text { Singapore } & 4 \\ \text { Slovenia } & 4 \\ \text { South Africa } & 4 \\ \text { Venezuela } & 4 \\ \text { Philippines } & 3 \\ \text { Poland } & 3 \\ \text { Bhutan } & 2 \\ \text { Ecuador } & 2 \\ \text { Nepal } & 2 \\ \text { Pakistan } & 2 \\ \text { Bangladesh } & 1 \\ \text { Belgium } & 1 \\ \text { Czechia } & 1 \\ \text { Denmark } & 1 \\ \text { Fiji } & 1 \\ \text { Finland } & 1 \\ \text { Italy } & 1 \\ \text { Lebanon } & 1 \\ \text { New Caledonia } & 1 \\ \text { Switzerland } & 1 \\ \text { Tunisia } & 1 \\ & \end{aligned}$

